\title{
UNCLASSIFIED
}

New York University

Courant Institute of Mathematical Sciences

Magneto-Fluid Dynamics Division

MF-125

DOE/ER/53223-196

\section{A SURVEY OF PROBLEMS IN DIVERTOR AND EDGE PLASMA THEORY}

Divertor and Edge Plasma Theory Working Group

U.S. Department of Energy

Grant No. DE-FG02-86ER53223

December 1992

\section{UNCLASSIFIED}

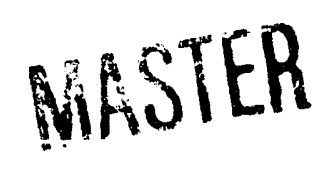




\section{DISCLAIMER}

This report was prepared as an account of work sponsored by an agency of the United States Government. Neither the United States Government nor any agency thereof, nor any of their employees, makes any warranty, express or implied, or assumes any legal liability or responsibility for the accuracy, completeness, or usefulness of any information, apparatus, product, or process disclosed, or represents that its use would not infringe privately owned rights. Reference herein to any specific commercial product, process, or service by trade name, trademark, manufacturer, or otherwise, does not necessarily constitute or imply its endorsement, recommendation, or favoring by the United States Government or any agency thereof. The views and opinions of authors expressed herein do not necessarily state or reflect those of the United States Government or any agency thereof.

Printed in U.S.A.

Available from

National Technical Information Service

U.S. Department of Commerce 5285 Port Royal Road

Springfield, VA 22161 
A Survey of Problems in Divertor and Edge Plasma Theory

December 22, 1992

Prepared by the Divertor and Edge Plasma Theory Working Group
A. Boozer, College of Wm. \& Mary
B. Braams, CIMS, NYU
R. Cohen, LLNL
R. Hazeltine, IFS, U. Texas
F. Hinton, GA
W. Houlberg, ORNL
E. Oktay, OFE, ex officio
D. Post, PPPL
W. Sadowski, OFE, ex officio
D. Sigmar, MIT
A. Wootton, PFC, U. Texas
H. Weitzner, CIMS, NYU, Chairman 


\section{TARLE OF CONTENTS}

Page

I.

Introduction

1

II. Fluid Models and Their Numerical Realization

III. Time Dependent Problems

11

IV. Non-Axisymmetric Effects

12

V. Anomalous Transport in the Scrape-Off Layer

14.

v. Edge Kinetic Theory

21

viI. Sheath Effects and Boundary Conditions in Divertors

27

VII. Electric Field Effects

31

IX. Atomic and Molecular Data Issues 34

X. Impurity Transport in the Divertor Region

XI. Poloidally Localized Power Dissipation

(MARPEs and Dense Gas Targets)

XII. Hellum Ash Removal

XIII. Neutral Transport

XIV. Selected Problems

XV. Review Papers on Edge Physics 


\section{INTRODUCTION}

On the bases of expected fusion energy production in a fusion reactor and of the energy and particle confinement in tokamaks as presently observed and understood, it is clear that a major design problem for a tokamak reactor is the development of a technique to accept the huge heat and particle fluxes at the first wall. At the same time there should not be a significant impurity reflux into the device. The introduction of a divertor is considered the best solution to the problem of absorbing the fluxes leaving the tokamak while maintaining a pure plasma. The ITER CDA has identified the divertor design as "one of the most difficult design tasks". The divertor design has also been a major issue for BPX-CIT and TPX. The studies of DEMO and tokamak reactors also identify the divertor action as a critical item for successful tokamak operation.

Given the importance of the divertor, it is no surprise that some time ago major efforts were established to model the plasma outside the separatrix. Typically these codes, of which many exist worldwide, follow the plasma, heat flows, neutrals and impurities in the region from the separatrix to the divertor plates. The system is often conceptually divided into a scrape-off layer adjacent to the core plasma and the somewhat distinct divertor region. These codes demand information from more or less standard fusion plasma physics, the physics of sheaths, and extensive data on atomic and molecular processes and surface physics. The experimental database on the scrape-off layer and divertor is presently rather limited, but in the near future extensive experimental information to compare against model predictions will become available.

Since the number of theoretical plasma physicists who have worked in this area is none too large, and in consideration of the increasing awareness of the importance of the topic and the growing possibility of comparison of experiment, theory and modelling, a modest refocussing of effort into this area would be highly desirable. Divertor and edge physics control and understanding are essential for ITER, for DEMO 
and for a reactor. The problems will not disappear when an ITER divertor design is committed. There is a need for a steady, continued, active presence of theoretical plasma physicists in this topic area.

Theoretical plasma physicists can help in the improvement of the physical models embodied in the existing codes, in exploring phenomena largely omitted in these codes, and in working with experimentalists to compare results and to propose relevant experimental tests. Any code has a complex set of assumptions built in, and in the process of comparison with data one should be open to the possibility of modification of modelling assumptions instead of the adjustment of an adjustable parameter. Theoreticians should be useful in such tasks.

The Divertor and Edge Physics Theory Working Group met in Dallas, Texas, December 2-3, 1992. We heard presentations from B. Lipschultz, MIT, B. Braams, NYU, and R. Stambaugh, GA. As a first step in enlarging and enhancing the effort in this topic area, we decided that a report describing the theoretical physics problems in the field might be of some use. The members of the committee, with consultation with some of their colleagues, subsequently prepared the following material.

Figure 1 presents a schematic drawing of a divertor and lists many problems related to divertor design. The problems are organized by the region in which they occur. In this report we present a description of some of the open questions in edge physics from a theoretician's point of view. Many of these topics in edge physics are likely to be of considerable importance. No doubt, some of them will ultimately be discovered to be largely irrelevant, while other issues not presently imagined may come to the fore. In addition to the background discussion of the topic, we present at the end a summary of a smaller number of issues that may have nearer term impact. We believe, given the long term nature of the problem, that all the issues need study, and not just the short term, high visibility topics. We hope that this survey will support those already working in this field and encourage others to work in the area.

The organization of the report is as follows. 


\section{Impurity Atomic}

Physics

Ionization

Recombination

Impurity Radiation

Emission

CX Recombination

Divertor Physics Issues for Conventional and Innovative Divertors

Gas Target Charge

Exchange, Radiation, Plasma Neutral Gas Interactions

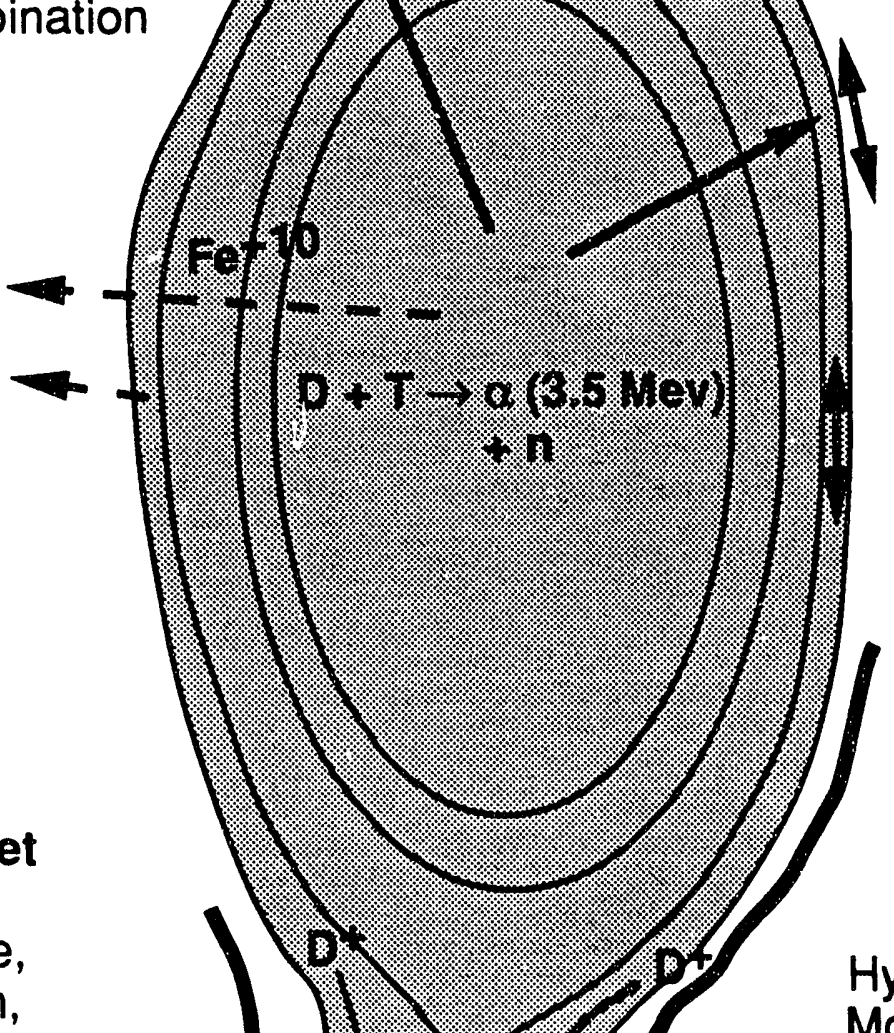

Neutral-Plasma Interaction

Charge Exchange Ionization

Recombination Impurity and Energy

Transport

Classical Transport

Anomalous Transport

Drifts

Electric Fields

Currents

Error Fields

Transport near $X$ point

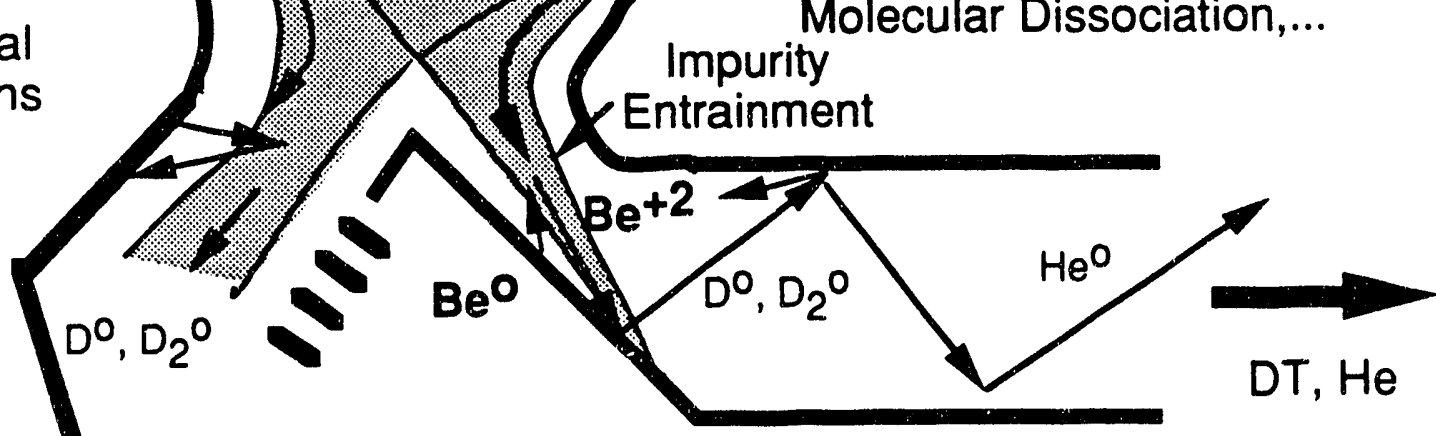

Materials issues

Thermal stress

Thermal conduction

$H$ implantation and permeation

Radiation damage

He formation

Surface Impurity Neutral Gas Transport Issues

issues

Sputtering

Erosion/Redeposition

Sublimation

Radiation Enhanced

Sublimation
Reflection

Absorption

Desorption

Thermalization

Neutral-Neutral Reactions 
Since the results of the codes form much of the basis for our understanding of the topics, the next section provides a cursory sketch of the models underlying the codes. The discussion also includes a description of some problem areas in the codes. These models are essentially all fluid representations of axisymmetric plasmas, which are usually assumed in steady state. In the next two sections we mention possibly significant time dependent and non-axisymmetric effects. A discussion of edge turbulence, another phenomenon lying outside the rnodels, follows. Kinetic theory provides possibilities for improving the fluid models and is discussed next. Sheaths and correct boundary conditions are assumed in the models, but many critical questions remain open, and are treated next. It is now generally accepted that electric field effects can modify the behavior of divertors significantly and can also modify ti.e code results dramatically, and we explore these issues. Some of the effects of the electric fields might readily be incorporated in existing models and codes; other issues require major reconsideration of the models. Impurity effects are critical to scrape-off layer and divertor problems. We consider three different topics in the area. The atomic and molecular physics data base must be organized and formatted in a way as to be useful to code developers and modelers. The needs in this area will be sketched. Beyond data, satisfactory models for transport of impurities must be developed, possibly equivalent to the "reduced charge state" theory of Hirshman and Sigmar. We add to this discussion an example of the possibly helpful effects of impurities in radiating energy in a manner similar to the effects of a MARFE. A discussion of the unique requirements for this removal of lielium ash produced in the plasma follows. A brief examination of neutral transport questions concludes the description of problem areas. The subsequent section provides a summary of selected problems of particular significance. The last section contains a brief bibliography of survey articles and related conference proceedings. 


\section{FLUID MODELS AND THEIR NUMERICAL REALIZATION}

Fluid models of the edge plasma have the form of the Navier-Stokes equations with certain twists and complications. The Navier-Stokes equations (in variables $n$, $u, T)$ are in any case nonlinear because of terms of the form $n u, n u u$, and $n u T$. In the plasma physics context the transport coefficients and source terms depend nonlinearly on temperature and density. The source terms for the plasma equations involve neutral gas recycling; with use of a fluid model for the neutral species this is just another coupled local transport equation, but if a Monte Carlo code is used for the neutrals then their contribution to the plasma equations introduces a nonlocal coupling. With the inclusion of several atomic species the equations take on aspects of chemically reacting flow; the ionization process defines the shortest reaction timescale, and the rate coefficients for ionization are strong functions of the electron temperature in the range of $T_{e}$ below the ionization level. In the bulk of the scrape-off plasma the typical flow velocity is well subsonic and the fluid is effectively incompressible, but the boundary conditions impose sonic flow, and a supersonic flow region plus shock is also often found in the calculations. The equations are highly anisotropic; when $T_{e}$ is a few hundred $\mathrm{eV}$, the ratio of parallel (classical) to perpendicular (anomalous) electron thermal diffusivity is about $1 \times 10^{9}$. A further anisotropy is provided by the diamagnetic terms in the transport equations. In a single calculation the temperature will vary over about two orders of magnitude and the transport coefficients over abou'c five. Thermal coupling between electrons and ions will be weak in some parts of the computational domain and very strong in others.

As a rough guide to some of the timescales relevant for edge plasma computations for typical large tokamak parameters: the shortest time is set by electron parallel thermal conduction across one cell: $\simeq 10^{-9} \mathrm{~s}$. Other short timescales are set by classical ion thermal conduction across one cell $\left(\simeq \mathrm{s}^{-7} \mathrm{~s}\right.$ before any flux limiting) and by ion sound across one cell $\left(\simeq 10^{-6} \mathrm{~s}\right)$. At the other end, th $\simeq$ radial temperature 
profile may get established in $\simeq 10^{-3} \mathrm{~s}$ and the slowest process is the establishment of a radial density profile in the very high recycling regime: $\simeq 10^{-1} \mathrm{~s}$, and it might not even be stationary.

There are about two dozen separate efforts on fluid modelling of the edge plasma in two-dimensional geometry, with a large variation in numerical approaches and a wide variation in the degree of success (these two variations are not necessarily correlated).

\section{Geometry}

The large ratio of parallel to perpendicular transport coefficients makes it desirable to employ a mesh that employs flux surfaces as one set of coordinates; on the other hand, in order to have enough geometric flexibility near the boundary structures it is desirable not to restrict the mesh to follow flux surfaces. Ease of discretization favors a smoothly varying mesh that is locally quadrilateral, whereas geometric flexibility favors unstructured triangles. A logically rectangular indexing scheme is convenient and contributes to fast execation, but a solution-adaptive mesh is more conveniently based either on unstructured triangles or on locally refined quadrilaterals.

A state-of-the-art edge plasma code could be based on either of the two geometric approaches just mentioned: unstructured triangles or locally refined quadrilaterals, in each case implemented in a solution-adaptive manner. In such a code, the discretization and solution algorithms would not assume that cell boundaries or mesh coordinates follow the flux surfaces, yet the code that generates the mesh would strongly favor local alignment with the magnetic geometry.

\section{Time Discretization}

It is widely agreed in the field of boundary plasma simulation that the timescale set by electron, or even ion, thermal conduction is too short to be admitted as a timestep limitation, so a successful code must be implicit at least for some components of the system of equations. One has a legitimate design choice, however, in whether or not to accept at the outset the ion sound timescale as a timestep limitation. Thus, 
one can isolate the heat conduction equations and the fast atomic processes and treat these implicitly in the context of an otherwise explicit marching scheme; alternatively one can apply backward differentiation in time to the system as a whole and attempt to work with timesteps significantly longer than the ion sound time, or even to work directly with the steady state equations. The trade-off depends on the cost per timestep (which can be significantly less in a marching scheme than in a fully implicit method), on any effective timestep limitation for a fully implicit method, and on the accuracy with which one attempts to follow the time evolution.

\section{Spatial Discretization}

Most computational fluid dynamics is based on the finite-volume approach, with the advantage that the treatment of advection-dominated flow is rather well understood. Within the finite-volume approach one still has an important design choice to make concerning the allocation of unknowns to cell centers, faces, and vertices. In the context of transonic flow the most common choice is to discretize all variables at cell centers, while in the context of incompressible flow the more common choice is to use a staggered mesh with velocities discretized at faces. The staggered mesh makes it easy to avoid the checkerboard pressure instability, which is a problem particularly in low-speed flow calculations. Having all variables at cell centers is more convenient, however, for evaluating the source terms. Also, if velocity along the magnetic field is to be treated differently from velocity across the field, and if the mesh is not aligned with the field, then there is no obvious way of assigning parallel velocity to any particular class of cell faces.

Some implementations of th finite volume method have the scalar variables, or all variables, assigned to cell vertices instead of cell centers. It could be very attractive to apply that style of discretization to the edge plasma equations because it offers the most compact representation of the diamagnetic fluxes.

Finite element discretizations are less common in the treatment of convection dominated equations. The classical Galerkin approach is unstable, but there exist 
various upwind-weighted variants. The Taylor-Galerkin method of Donea is coming to be widely used for time dependent finite element flow simulations, and is also being used for edge plasma simulation.

\section{Solution Algorithms}

Many different methods are used in practice to solve the large systems of equations that arise from the implicit discretization of the evolution equations. The three main categories are (1) dimensional splitting, which leads to dense banded linear equations for all physical variables along a single mesh line; (2) a segregated variables approach that leads to sparse two-dimensional equations governing a single physical variable on the whole domain; and (3) a complete coupled equations approach, in which a matrix equation is set up and solved (e.g. with use of some form of non-symmetric ILU/CG iteration) that couples each variable on each c.? to all variables on the same cell and its four or eight neighbour cells.

Per iteration, the first two methods require much less time than does the third, but they also require many more iterations. A code that relies on a more approximate cheap solution algorithm can afford to use simple approximations to the Jacobian, whereas a code that requires at each iteration the solution of a large linear system must ensure that an accurate Jacobian is employed. We observe that the codes that rely on dimensional splitting or on segregated variables invariably choose to employ rather easily constructed approximate Jacobians (assuming, e.g., frozen transport coefficients), whereas the codes that employ the full Jacobian rely on numerical differentiation to construct the most accurate linearized system.

All these solution algorithms are viable, and the success of any particular method depends much on the skill with which it has been implemented. A sensible comparison must consider specific codes, rather than the methods in the abstract, and must also to some extent consider specific problem classes (e.g., accurate evolution calculations or steady or quasi-steady states). 


\section{References for Numerical Issues:}

References $[1,2]$ are survey articles on plasma edge modelling. References [3-7] provide textbook treatment of relevant computational fluid dynamics. The remaining papers concern issues that are not adequately discussed in textbooks. References $[8,9]$ present conjugate-gradient-like iterative methods for nonsymmetric linear equations. Reference [10] presents the Taylor-Galerkin finite element method, and Reference [11] concerns the use of an unstructured adaptive triangular mesh in Navier-Stokes computation.

1. E.L. Vold, Multidimensional and Multifluid Plasma Edge Modelling: Status and New Directions, presented at the Plasma Edge Theory Workshop, Bad Honnef, Germany, June 1992; to appear in Contrib. Plasma Phys.

2. D. Reiter, Progress in 2-Dimensional Plasma Edge Modelling, presented at the 10th International Conference on Plasma-Surface Interactions, March 1992; to appear in J. Nucl. Mater.

3. R.D. Richtmyer and K.W. Morton, Difference Methods forr Initial Value Problems, 2nd edition, Wiley, New York, 1967.

4. D.A. Anderson, J.C. Tannehill and R.H. Pletcher, Computational Fluid Mechanics and Heat Transfer, Hemisphere/McGraw-Hill, New York, 1984.

5. C.A.J. Fletcher, Computational Techniques for Fluid Dynamics (Vol. 1: Fundamental and General Techniques, Vol. 2: Specific Techniques for Different Flow Categories), Springer Verlag, Berlin, 1990.

6. R.J. LeVeque, Numerical Methods for Conservation Laws, Birkhauser, Boston, 1990.

7. von Karman Institute for Fluid Dynamics, Lecture Notes on Computational Fluid Dynamics, annually. 
8. Y. Saad and M.H. Schultz, Conjugate Gradient-Like Algorithms for Solving Nonsymmetric Linear Systems, Math. Computation 44 (1985) 417-424.

9. R.W. Freund and N.M. Nachtigal, QMR: A Quasi-Minimal Residual Method for Non-Hermitian Linear Systems, Numerische Mathematik 60 (1991) 315-339.

10. J. Donea, A. Taylor-Galerkin Method for Convective Transport Problems, Int. J. Numer. Methods Eng. 20 (1984) 101-119.

11. R. Loehner, K. Morgan, J. Peraire and M. Vahdati, Finite Element FluxCorrected Transport for the Euler a:dd Navier-Stokes Equations, in Finite Elements in Fluids, Vol. 7, edited by H. Gallagher et al., W'iley, New York, 1987. 


\section{TIME DEPENDENT PROBLEMS}

The heat lnad to the divertor is increased considerably for short periods of time by disruptions. These must be allowed for in the design of the divertor, since they are a ubiquitows ature of tokamak operation. Another time dependent phenomenon of some impuitance is the occurence of ELMs.

\section{Physics of ELMs}

Periodic oscillations associated with instabilities, especially those localized near the plasma edge, i.e. ELMs, make the heat load time dependent [E.J. Doyle et al., Phys. Fluids B3, 2300 (1991)]. ITER will probably operate in $\mathrm{H}$-mode with frequent ELMs. Under these conditions, a non-negligible fraction of the heat exhaust is in the form of pulses which last a few milliseconds. The duty cycle of these pulses affects the peak heat loads. Also, ELMs are potentially very useful in limiting the plasma density and the impurity influx. They can therefore influence the access to radiative divertor designs. They need to be included in edge transport modelling studies. Some types of ELMs are thought to be related to ideal ballooning modes, but the relation needs to be clarified. Also, there is a type of ELM which occurs well below the ballooning mode instability threshold, which must have a different mechanism. These are presently not understood. A better basic understanding of ELMs is needed before a divertor design is complete. It, would also be desirable to develop some ideas about how to control them, in order to control impurities and the rise in density in $\mathrm{H}$-mode. 


\section{NON-AXISYMMETRIC EFFECTS}

\section{Ripple Transport}

Ripple transport could affect the loss of energetic ions near the plasma edge. If these contribute significantly to the divertor heat load, or if the average energy of such ions exceeds sputtering thresholds for the divertor surfaces, then it could set acceptable limits on toroidal field ripple. This must be determined before the toroidal field coil design is completed.

\section{Transport Effects of MHD Modes or Islands Near the Plasma Edge}

MHD modes or islands near the plasma separatrix may affect the energetic ions in ways similar to ripple transport. It could impact heat loads and sputtering rates in the same way. Since MHD modes may be difficult to eliminate, their effects must be determined and allowed for, as part of the divertor design.

\section{Stochastic Divertor and Ergodic Magnetic Field Transport}

Magnetic perturbations that arise naturally, or that are intentionally introduced by perturbing coils, move the last closed magnetic surface inside the separatrix of the axisymmetric tokamak. ${ }^{1-6}$ Field lines started just outside the last closed surface sample the region between the last closed surface and the axisymmetric separatrix in a complicated, quasi-stochastic manner. ${ }^{3,6}$ This stochastic layer presumäbly has high transport due to complicated electric fields across the magnetic field lines as well as flow along the magnetic field. The stochastic layer could greatly modify the plasma dynamics of the edge, particularly that of the electrons. This layer could be used to broaden the heat load in the electron channel, thus reducing the peak heat load on the divertor plates. It might also provide enhanced shielding of the bulk plasma from impurities. Self-consistent calculation of the electrostatic potential is also needed in the region of stochastic field lines. Magnetic perturbations also cause field lines started near the last closed magnetic surface to intercept the divertor plates in strips rather than along a circle as in an axisymmetric divertor. ${ }^{5,6}$ The magnetic flux contained 
in these strips is almost an order of magnitude smaller than that contained in the stochastic region between the last closed surface and the axisymmetric separatrix.

The effects of magnetic perturbations on divertor theory and on the H-mode transition have received only limited attention. Some level of perturbations is intrinsic to tokamaks and must be included in a realistic theory of the divertor. Potentially beneficial effects of intentional magnetic perturbations, such as spreading the heat load on the divertor plates and providing enhanced shielding against impurities require quantification.

\section{References:}

1. Y. Tomita, S. Seki and H. Momota, J. Phys. Soc. Jpn. 42, 687 (1977).

2. A. Boozer and A. Rechester, Phys. Fluids 21, 682 (1978).

3. D. Skinner, T. Osborne, S. Prager and W. Park, Phys. Fluids 30, 1218 (1987).

4. R.J. LaHaye, Nucl. Fusion s, $\$ 550$ (1991).

5. N. Pomphrey and A. Rieman, Phys. Fluids B 4, 938 (1992).

6. A. Punjabi, A. Verma and A. Boozer, Phys. Rev. Lett. 69, 3322 (1992). 


\section{ANOMALOUS TRANSPORT IN THE SCRAPE-OFF LAYER}

Anomalous (turbulent) transport in the scrape-off layer SOL has received relatively little attention throughout the world. This is true both theoretically and (with a few notable exceptions such as TEXT and ASDEX) experimentally. "Relatively" here applies both with respect to anomalous transport in other parts of the tokamak and with respect to other aspects of the SOL. Typically, anomalous transport is handled in the 2-D fluid modeling codes by prescribing fixed numbers for anomalous transport coefficients. Yet the level of anomalous transport clearly plays a criticial role in establishing the SOL thickness, and may well play a significant role in determining the performance of advanced divertor concepts such as radiative and gas-target divertors.

In contrast, anomalous transport in the closed-flux-surface "edge" region has been the subject of considerable investigation for the past several years, largely because of its likely connection to the difference in confinement between L-and $\mathrm{H}$-modes and its possible involvement in the $\mathrm{L}-\mathrm{H}$ transition. The SOL and edge can not be treated separately; most likely turbulence generated in one region affects the other. We will concentrate here on turbulence generated in the SOL, as this is the area in greater need of additional effort.

\section{Status}

There are several drives for instability unique to the SOL open-field-line geometry. These include curvature-driven modes ${ }^{1,2}$ destabilized by incomplete line tying through the sheath, modes driven by rotation of the plasma relative to the conducting end wall $^{3,4}$ (these have been described as electron-temperature-gradient driven modes; they will be referred to here as the conducting-wall-driven modes), and modes driven by parallel gradients of the $\mathbf{E} \times \mathbf{B} \mathrm{drift}^{5}$ within the plasma. Cross-field variation of $\mathbf{v}_{E \times B}$ can drive a Kelvin-Helmholtz instability, but the effect is apparently only significant within a few gyroradii of a wall which is tangent to field lines, where a 
radial sheath develops. ${ }^{6}$ In addition, there are drives local to the edge but not specific to the SOL, such as radiation ${ }^{7}$ and ionization, ${ }^{8}$ and possible SOL versions of modes which also exist in the core, such as ion-temperature-gradient-driven modes.

The SOL-specific drives generate strong instabilities, and the $\chi$ 's predicted by mixing-length estimates appear to be roughly consistent with experiments. For typical parameters the maximum growth rate with only the conducting-wall drive is larger than that with only the curvature drive; a similar comparison for the axial shear mode häs not been done, but a rough estimate indicates comparable growth rates. Hence, all three drives may have a significant role in SOL transport. There is analytic work on these modes by a collaboration involving U.S. institutions (LLNL, IFS, UCSD, UCB) and Russia. Work on the curvature-driven modes has been done in Europe and Russia. The work to date has been mainly linear theory (growth rates, stability boundaries) and simple (mixing-length) transport estimates. The work has been largely in a radially-local approximation, though some work has been done $e^{9,10}$ on the coupling of SOL and edge regions.

For standard SOL operation, the ionization-driven instability as described in Ref. [7] is weaker than the SOL-specific modes. The radiation-driven instability, as described in Ref. [8], could be quite significant; however, as noted in Ref. [8], the drive is "fickle", operative only if the radiation rate is a decreasing function of temperature at the operating point. Hence radiation-driven turbulence would be expected to be strong under some operating conditions and absent under others. Furthermore, the theory needs to be reassessed for the open field-line geometry of the SOL. For the radiative and gas-target divertor scenarios, the radiative and ionization drives would be expected to play larger roles. Hence, assessment of radiative and ionization-driven turbulence in SOL's is an important outstanding problem.

The angle of incidence of field lines on divertor plates can be small, for instance one degree in DIII-D. Hence a radial sheath can develop, and the Kelvin-Helmholtz instability may be locally important. The signifcance of the resultant turbulence for 
a realistic tokamak configuration has not been determined.

We are not aware of any work on the effect of turbulence on parallel flows in the SOL. Yet there is at least one piece of evidence suggesting a significant effect: for DIII-D experiments in which divertors are biased to alter the distribution of particles and power on the divertor plates, the experiment shows smaller shifts and a smaller induced current than 2-D (LEDGE code) calculations using Spitzer resistivity. ${ }^{9}$

A major complicating factor in SOL turbulence is the lack of separation of fluctuation and equilibrium scales. The fluid displacements which follow from mixing-length estimates are comparable with observed SOL widths, ${ }^{11}$ and there are theoretical, ${ }^{12}$ experimental ${ }^{13,14}$ and computational ${ }^{15}$ indications that wave energy preferentially inverse cascades to long length scales. These observations cast doubt on the applicability to the SOL of the usual renormalized turublence theory approaches, and also on the utility of the diffusive transport approximations which are basic to the present SOL modeling codes. Reference 12 suggests an alternative to both, namely a variational calculation of a minimum-enstrophy state (similar to the minimum-energy Taylor state for a RFP), and carries out the calculation for a simple model limiter geometry.

To our knowledge, there has been (only) one serious attempt at numerical simulation of turbulence in an open-field line SOL, that of $\mathrm{Xu}^{15}$; so far it has only been run as a $2 \mathrm{D}$ code (but is formulated as $3 \mathrm{D}$ ), and is limited by being electrostatic, fluid, and containing only the conducting-wall drive. This simulation has followed the linear and nonlinear phases of the flute version of the conducting-wall instability, has demonstrated saturation, and shown the expected dual-cascade behavior. There are other codes which may be useful; in particular, the Maryland group (Drake, Guzdar, McCarthy, et al.) has 3D electrostatic codes including a simple version of a limiter. With some upgrading of the limiter physics and the addition of a temperature equation, these codes could simulate the SOL modes. A code with similar capabilities is under development at the Oak Ridge National Laboratory. Finally, O. Pogutse has 
developed a $2 \mathrm{D}$ code which includes curvature drive and proper sheath physics, but does not yet evolve a temperature equation.

Like the theoretical work itself, comparison of SOL turbulence theory with experimental data is in its infancy, limited to noting some points of contact with data. Such points of contact have been noted for both the curvature-driven modes ${ }^{1,2}$ and the $\mathbf{E} \times \mathbf{B}$ modes. $^{3-5,11,12}$ Unfortunately there is relatively little data with which to compare, since SOL turbulence measurements have not been a high priority, particularly on large diverted tokamaks. (Even on relatively well-diagnosed machines such as TEXT, the information from the SOL is limited.)

There has been a first effort at incorporating transport coefficients from a turbulence model (that of Ref. 4) in an SOL modeling code (LEDGE); Ref. 9 and papers presented at the 1992 APS Plasma Physics Meeting. 'The code effects such calculations, but no detailed comparisons with experimental profiles has been been attempted.

\section{Needs}

The list of needs follows immediately from the status given above. Probably the most urgent need is to identify the most likely sources for turbulence and estimate transport, and especially its scaling. The cases of "advanced divertors", such as gastarget and radiative divertor, are of particular interest. Further, attention should be given to assessing impurity-driven and ionization-driven turbulence in the open-ended SOL geometry.

Also of high importance is the benchmarking of predictions for the various turbulence models (for conventional and advanced divertors) with experimental data and the incorporation of the transport predictions from the models into the 2-D SOL modeling codes. Benchmarking must include both predictions of the theories themselves and testing the results from the SOL codes. As noted above, incorporation of theory-based transport coefficients has been undertaken for one model with the LEDGE code, but benchmarking is just beginning. Even though the radial trans- 
port may well be non-diffusive, incorporation of effective diffusion coefficients (which give the correct scale lengths) is a reasonable near-term approach for synthesizing a comprehensive SOL modeling capability.

There is also the need for more turbulence simvlation work (including $3 \mathrm{D}$, electromagnetic, curvature drive, equilibrium parallel current, atomic physics, and kinetic effects). An extended simulation capability will provide an additional means of benchmarking analytic predictions as well as giving predictive tools in their own right. Given the unclear separation of scales, a 3D nonlinear simulation may ultimately be the best way to model boith the equilibrium and the fluctuations in the SOL.

Other needs include: linear theory for the various drives with nore realistic geometry; linear theory for the mode with parallel shear in $\mathbf{v}_{E \times B}$ at a level comparable to that done already for the other SOL modes; calculations of radial mode structures (important since $k_{\perp}$ times the radial temperature-gradient scale length is not large), a careful look at the temperature response for non-flute perturbations (present analysis is based on reduced ideal MHD, which may be inadequate); nonlinear calculations of saturation and transport; and of extension of the minimum-enstrophy calculation to realistic (2D equilibrium) geometry and inclusion of atomic physics. In developing nonlinear calculations, it will be particularly important to quantify separately the energy transport of electrons and ions, as they can be energetically decoupled and play distinct roles in the divertor heat load problem.

It was noted above that there has been relatively more work done on the closedflux-surface "edge" region. This work includes investigation of the role of turbulent Reynolds stress in establishing the electric-field. Such calculations should be revisited in light of the likely penetration of SOL-generated modes into the edge. Also, this approach to determining the electric field has not made its way into the SOL modeling codes (even though the computational domain of such codes includes the "edge" region); this situation should be remedied. 


\section{References}

1. A.V. Nedospasov, Fiz. Plazmy 15, 1139 (1989) [Sov. J. Plasma Phys. 15, 659 (1989)].

2. X. Garbet, L. Laurent, J.-P. Roubin and A. Samain, Nucl. Fusion 31, 967 (1991).

3. H.L. Berk, D.D. Ryutov and Yu. Tsidulko, Phys. Fluids B 3, 1346 (1991).

4. H.L. Berk, R.H. Cohen, D.D. Ryutov, Yu. Tsidulko and X.Q. Xu, Nucl. Fusion (in press).

5. Yu. Tsidulko, H.L. Berk and R.H. Cohen, 1992 APS-DPP Meeting, and paper to be submitted to Phys. Fluids.

6. K. Theihaber and C.K. Birdsall, Phys. Fluids B 1, 2244 (1989), and Phys. Fluids B 1, 2260 (1989); S.E. Parker, X.Q. Xu, A.J. Lichtengerg and C.K. Birdsall, Phys. Rev. A 45, 3949 (1992).

7. See e.g. D.R. Thayer and P.H. Diamond, Phys. Rev. Lett. 65, 2784 (1990).

8. See e.g. A.S. Ware, P.H. Diamond, H. Biglari, B.A. Carreras, L.A. Charleton, J.N. Leboeuf and A.J. Wootton, Phys. Fluids B 4, 872 (1992).

9. R.H. Cohen, S.L. Allen, H.L. Berk, et al., 1992 IAEA Meeting, Paper IAEACN-56/D-1-3.

10. X.Q. Xu, M.N. Rosenbluth, P.H. Diamond, at 1992 Sherwood Theory Meeting.

11. R.H. Cohen, X.Q. Xu and B.M. Modi, at 1992 APS-DPP Meeting.

12. N. Mattor and R.H. Cohen, submitted to Phys. Fluids. 
13. As indicated by the spectra in J.G. Watkins, R.A. Moyer, D.N. Hill, D. Buchenauer, T.N. Carlstrom, R. Conn, J. Cuthbertson, R. Doerner, R. Lehmer, M.A. Mahdavi, R.T. McGrath, L. Schmitz, and R. Stambaugh, J. Nucl. Materials, Proceedings of 10th International Conference on Plasma Surface Interactions, 1992 (to be published).

14. Ch.P. Ritz, E.J. Powers and R.D. Bengston, Phys. Fluids B 1, 153 (1989).

15. X.Q. Xu, C.K. Birdsall, M.N. Rosenbluth and P.H. Diamond, at 1992 APS-DPP Meeting. 


\section{EDGE KINETIC THEORY}

\section{Role of Kinetic Theory in Edge Physics}

Kinetic analysis of the plasma edge has two objectives. First, one solves an appropriate kinetic equation to relate various particle, momentum and heat fluxes to the gradients of densities, velocities and pressures - the conventional program of transport theory. In this regard kinetic theory acts as a closure vehicle for systems of moment or fluid equations. The second application is more specific to the tokamak edge: to study effects associated with strongly non-Maxwellian distributions, as resulting, for example, from prompt particle losses or potent particle and energy sources.

\section{Closure}

Transport perpendicular to the magnetic field is a relatively straightforward issue, even at long mean free path, because the gyroradius serves to localize trajectories on flux surfaces. In the scrape-off layer (SOL), however, one is at least equally concerned with parallel transport, and at long mean free path, parallel transport need not be local, in the sense of depending linearly on the local gradients. One strategy for this case is now under exploratory study: one relates the parallel energy flux, for example, to a blurred temperature gradient, averaged over an appropriate length along the field line. However the conventional treatment, employing so-called flux limits, is much simpler: one notes that, in the long mean free path limit, energy transport is bounded by parallel streaming, suggesting the limiting heat flux.

$$
q_{\ell}=\alpha v_{t} n T
$$

where $v_{t}$ is the thermal speed and $\alpha$ is a numerical coefficient, of order one-tenth to a few tenths, that is used as a adjustable parameter. A simple interpolation formula, involving $q_{\ell}$ as well as the classical (short mean-free path) heat flux, is then presumed to describe heat transport over the interesting range of collisionalities. 
The flux-limiting procedure is evidently sensible, but hardly justified by physical argument. (For example, even if the relevance of $q_{\ell}$ is accepted as a limiting case, no unique interpolation prescription can be inferred.) Moreover it is found that the flux limitation formula can affect fluid model predictions in crucial ways. Thus analysis of long-mean-free path parallel transport, with the objective of a convincing transport description, would have critical impact on SOL modeling. Of course such an analysis would illuminate not only heat flux but all relevant parallel transport processes, including viscosity and the so-called "thermal force," a collisional momentum exchange process involving temperature gradients.

\section{Non-Maxwellian Distributions}

Transport theory provides an effective closure scheme only if the distribution functions of each species can be described by a small number of variables, such as the densities, temperatures and flow speeds. Such a reduced description generally requires the distribution be approximately Maxwellian. Core distributions are Maxwellian, even at very long mean free path, because core confinement times are long compared to the collision time; in the edge region, however, particles - especially the faster moving electrons - can be lost and replaced too rapidly for Maxwellian relaxation. The effect is to allow a number of non-Maxwellian distortions, even in lowest order: loss regions, as in mirror geometry, as well as velocity-space bulges associated with the various sources (such as ionization) peculiar to the edge region.

In the worst case one could attempt to solve the kinetic problem numerically: a Fokker-Planck or Monte Carlo code could in principle take over the present role of fluid codes in edge physics. Of course such a program would be extremely ambitious, testing the limits of code development and even computational hardware. More realistically, one might adopt a hybrid strategy, coupling a Monte Carlo description of electrons to a more conventional fluid description of ion species. It seems most likely, however, that analytical theory could play a role in studying non-Maxwellian effects analogous to its role in transport closure, providing enough information to allow a 
closed description of edge dynamics in coordinate space rather than phase space. We consider next the main components of such an analytical theory.

\section{Special Features of Kinetic Theory in the Edge Region}

We have mentioned several features that make edge kinetic theory distinctive. Here we list more systematically some critical and distinctive elements of kinetic theory near the tokamak edge. Of course the list cannot claim to be exhaustive. The discussion is brief because most of the following topics are also discussed, from different points of view, elsewhere in this report.

\section{Collisionless Orbits}

The phase space region corresponding to prompt losses in a diverted tokamak the tokamak loss region - can be determined only from detailed knowledge of particle orbits. Orbit analysis near the edge is distinctive for several reasons. There is first the proximity of plasma boundaries: the vacuum wall and divertor collector. Second, the magnetic separatrix, with 's small rotational transform and narrow chaotic layer, requires careful treatment (at least for electron motion). Finally and perhaps most importantly, the large radial electric fields predicted and observed near the edge can dramatically alter orbit geometry. Thus gradients in the electric field cause squeezing of banana orbits, while larger fields (even when radially constant) can yield such strong poloidal drifts as to undo magnetic trapping. Such orbit modification seems likely to play a role in fixing the self-consistent radial potential.

\section{Sources and Sinks}

The near steady state on a tokamak flux surface reflects, not only diffusional exchange with neighboring surfaces but also such local sources as radiative cooling and ionization local sources. Source terms in the edge region are exceptionally numerous 
and virulent: sputtering and other wall-reflux processes, impurity line radiation, and charge exchange have a pivotal effect on particle and, especially, energy budgets. The effect on kinetic theory is to introduce exchange terms into the Fokker-Planck equation for each species, thus coupling of the kinetic equations for several species, including charged and neutral particles.

\section{Charge Exchange}

Charge exchange (CX) with neutral hydrogen, while conserving particle numbers for each plasma species, yields an important energy and momentum loss mechanism. Since CX mean-free paths are very small in typical edge environments, the effect of neutrals is localized to regions close to the vacuum wall. There, however, CX can drastically affect energy transport as well as plasma mass motion. Indeed, the effectively stationary character of newly liberated neutrals provides a reference frame, unique to the edge, for plasma rotation. Theorists have sometimes dismissed such issues - the rubric "kitchen physics" is telling - but in fact the kinetic description of neutral particles can be elegant, ${ }^{1}$ and the coupling to ion dynamics is surprisingly simple. One consequence of $\mathrm{CX}$ momentum exchange requires particular assessment: the radial ion diffusion corresponding to CX friction is not intrinsically ambipolar, and therefore seems to affect the radial electric field.

\section{Space Charge}

Space charge, a traditional concern of fundamental plasma physics, receives little attention in studies of tokamak confinement. It is apparently thought that quasineutrality rules out significant charge imbalance, except within a Debye length of material walls. In fact the quasineutrality argument cuts both ways: while implying that the total charge density must be in a certain sense small, it also shows that even very small charge imbalance will drastically affect the electric field. Such is the case near the tokamak edge, where the experimentally observed sharp curvature (large secondderivatives with respect to radius) in the radial potential reveals exceptionally strong space charge. It presumably arises from unbalanced losses - from differences between 
ion and electron orbits. Thus more electrons can stream to collector plates (avoided by some ions due to their drifts across field lines), implying a potential peak, while the radial drift may cause ions to preferentially strike a container wall, yielding instead a

potential minimum. A space-charge explanation that treats collisionality, geometry and self-consistent electric-field in a convincing way is not available - and sorely needed. What is clear is that the resulting radial electric field has striking effects on tokamak confinement.

\section{Depleted Distribution Functions}

We have noted that loss regions and local source terms can give the edge-region distribution functions, especially the electron distribution, a strongly non-Maxwellian character. A credible picture of plasma behavior in the SOL, including flow rates to the divertor, space charge and the electric field profile near the separatrix, will require understanding these distributions. What seems to be called for, short of a full and daunting Fokker-Planck treatment, is a tokamak version of the classic mirror problem solved by Pastukov and others. The key feature, however idealized, is two-dimensional: radial diffusion across tise separatrix, followed by velocity diffusion and parallel streaming to a collector plate. (Note that radial motion and parallel motion here act in series, rather than in parallel, as occurs in the mirror case.) While considerable progress on this problem has been achieved, ${ }^{2}$ its complete solution and integration into fluid models requires much more effort.

\section{Steep Gradients}

Most of the topics listed above are affected by the exceptionally steep gradients - in plasma densities and temperatures, and especially in the radial potential commonly observed in the tokamak edge region. On the one hand, such gradients can be expected to drive various plasma instabilities, perhaps accounting for the large relative fluctuations in density and potential observed near the edge. Just as importantly, the gradients alter the kinetic description in a fundarnental way. The point is that conventional kinetic treatments, such as that of neoclassical theory, assume the 
poloidal gyroradius - the gyroradius measured in the poloidal magnetic field - to be small compared to any scale length of interest. Equivalently it is assumed that many particle orbit widths fit into any macroscopic gradient length. This assumption is challenged in the edge: observed radial electric fields, for example, correspond to potential scale length nearly comparable to the poloidal gyroradius. (The fact that the poloidal gyroradius is itself rather large near the separatrix contributes to this circumstance.) A convincing description of edge kinetics will have to address this breakdown in conventional orderings.

\section{References}

1. e.g. J.W. Connor, Plasma Phys. 19, 853 (1977).

2. F.L. Hinton and M.S. Chu, Nucl. Fusion 25, 345 (1985). 


\section{SHEATH EFFECTS AND BOUNDARY CONDITIONS IN DIVERTORS}

The boundary conditions at the divertor plate are most simply taken to be that the electrons and the ions are perfectly absorbed at an equal rate, corresponding to local ambipolarity. If one assumes the magnetic field lines are normal to the divertor plate, there is a jump in the electric potential ${ }^{1,2}$ across a Debye-length-scale sheath with $\Delta \phi \approx 3 T_{e} / e$. This jump in potential reflects all but the superthermal electrons and accelerates the ions. The electron distribution function is, therefore, a truncated Maxwellian. Collisions are essential for determining the ion distribution function near the sheath and sheath effects on the ion flow extend back a few ion mean free paths. The slowest flow that is consistent with a perfectly absorbing divertor plate ${ }^{3}$ is $v \geq\left[\left(T_{e}+\gamma_{\mathrm{eff}} T_{i}\right) / m_{i}\right]^{1 / 2}$ with $1 \leq \gamma_{\mathrm{eff}} \leq 5 / 3$. These boundary conditions imply that the electron energy flux into the divertor plate ${ }^{4}$ is $q_{e}=\alpha \eta T_{e} v$ with $\alpha \approx 5$ and the ion energy flux is $q_{i} \approx(5 / 2) n T_{i} v$.

The critical issues of the boundary conditions are of four types: effects of the non-zero mean free path of the electrons and ions, effects of a tangential component of the magnetic field on the sheath, the effects of the plate not being a perfect, ambipolar absorber, and the effects of neutrals, especially if the neutrals prevent hot plasma contact with the divertor plate. These effects not only change the boundary conditions of the simple divertor model, but also change the behavior of impurities, such as alpha particles.

The effects of a non-zero mean free path arise near the divertor plate at even lower plasma temperatures than in the overall scrape-off layer, due to the small spatial scale that is established by charge exchange process near the divertor plates. If one uses units of meters and electron volts the mean free path of electrons and ions is $\lambda \approx 10^{16} T^{2} / n$. The electron heat flux at the sheath, determined by the form of the distribution function at an energy of about $4 T_{e}$, is especially sensitive to the mean free path. The effects of the ions on the flow into the sheath always involve the mean 
free path and become very complicated if the ion mean free path is comparable to the shortest scale of the flow. In the simplest models of plasmas flowing into plates, one assumes $T_{i} \ll T_{e}$ to eliminate the subtle effects of ions on the flow.

If the magnetic field is almost tangential to the divertor plate ${ }^{5}$ the electric potential structure in the sheath becomes complicated. If the angle that the field makes with the plates is less than roughly $\left(m_{e} / m_{i}\right)^{1 / 2}$ radians, which is roughly $1^{0}$, the electric potential becomes positive relative to the plasma on the scale of an ion gyroradius. Closer to the plate, on the Debye length scale, the potential becomes negative relative to the plasma and the total jump in the potential from the main body of the plasma to the plate remains $\Delta \phi \approx-3 T_{e} / e$. The positive jump in the potential can hinder the removal of multiply ionized ions from the scrape-off layer.

Although the total outflow of ions and electrons to all the divertor plates must be approximately equal, it is not required that an equal number of electrons and ions flow to each divertor plate or to any particular area on a single divertor plate. ${ }^{5}$ The absence of local ambipolarity implies the flow of currents in the plasma and in external structures. The effects of these currents are important as are the effects of the changed boundary conditions on the main body of the plasma in the scrape-off layer.

A desirable feature of a divertor would be that the energy is radiated away rather than lost by flow into the divertor plates. If this type of divertor is achieved, the primary boundary conditions on the flow in the scrape-off layer are set by the hot plasma interacting with neutrals. This interaction has a number of natural scales involving atomic processes and therefore gives more complicated conditions.

Boundary conditions supplied by the sheath determine many of the basic properties of the scrape-off layer and many of the effects of the plasma on the divertor plates. The boundary conditions on the flow speed and the ion energy flux are dependent on effects that occur over an ion mean free path. Model calculations should be done to better determine reasonable assumptions for these boundary conditions. 
In tokamaks, the divertor plates intercept the magnetic field lines at a very small angle $\theta_{p}$. A magnetic presheath exists along the field lines for a distance $\rho_{i} / \sin \theta_{p}$ with $\rho_{i}$ the ion gyroradius. The primary work in this area has been in the fluid approximation. In practice the collisional effects can arise on a scale comparable to that of the magnetic presheath. Unless the electron mean free path is short compared to the characteristic spatial scales of the divertor, the tail of the electron distribution function will be significantly modified from the form assumed in sheath calculations. These modifications can either enhance or reduce the flux of energy in the electron channel. The shortest spatial scale in divertor problems is often set by atomic physics processes. The boundary conditions on a plasma intercepting a neutral gas must be clarified due to the desirability of a radiative divertor.

\section{References}

1. L. Tonks and I. Langmuir, Phys. Rev. 34, 876 (1929).

2. G.A. Emmert, R.M. Wieland, A.T. Mense and J.N. Davidson, Phys. Fluids 23, 803 (1980).

3. D. Bohm, in The Characteristics of Electrical Discharges in Magnetic Fields, edited by A. Gutherie and R.K. Wakerling (McGraw-Hill, New York, 1949), Ch. 3, p. 77.

4. G.D. Hobbs and J.A. Wesson, Plasma Physics 9, 85 (1967).

5. R. Chadura, Phys. Fluids 25, 1828 (1982).

6. A.H. Boozer, Phys. Fluids 19, 1210 (1976).

\section{Structure of the Presheath}

A rather old but still open issue is the structure of the presheath, especially the variation of electric potential across it. The presheath consists of the region extending 
along a field line from the outer edge of the Bohm sheath into and throughout the region of significant ionization. A common prescription for the potential drop across the presheath is that it is whatever is necessary to accelerate ions to their sound speed at the edge of the sheath. Various definitions of the sound speed are used. The basis for this formula rests on calculations with cold ions, a uniform electron temperature, and a localized source [as in Self and Ewald, Phys. Fluids 9, 2486 (1966)]. The prescription has a number of shortcomings, such as being ill-defined for multiple ion species. The 2D fluid codes calculate a presheath structure, but the calculations are based on the standard short-mean-free-path fluid equations, whereas the mean free path can easily be comparable to the short scale length of the neutrals. There is an ongoing effort to do analytic and numerical kinetic modeling. More effort is needed, aimed at both the development of analytic formulas for the presheath potential drop and at improved descriptions for the SOL codes. 


\section{ELECTRIC FIELD EFFECTS}

First principles based fluid (and kinetic) models for the electric field in divertors and edge plasmas need to be developed to gain insight and identify the dominant processes that both determine and allow control of the self-consistent electric field and its effect on particle and heat exhaust.

Parallel flow along the magnetic field lines to the divertor plates alters the ambipolarity of tokamaks by introducing an end loss modification of the continuity equation. Moreover, the radial and parallel flows $\langle N \mathbf{V} \cdot \nabla \psi\rangle$ and $N \mathbf{V}_{\|}$depend on the electric field in different ways. In addition, $N \mathbf{V}_{\|}$depends on the potential barrier created at the divertor plates to confine electrons. In the core, conservation of toroidal angular momentum is normally employed to evaluate the radial particle flux $\langle N \mathrm{~V} \cdot \nabla \psi\rangle$ in the absence of end loss. Consequently, a new parallel momentum flow term must be retained outside the separatrix.

The self-consistent electric field established beyond the separatrix depends on whether the divertor plates are insulators or either unbiased or biased conductors. Except for the case of insulating divertor plates, ambipolarity no longer requires that the radial ion flux be equal to that of the electrons. Instead it can be balanced in part by electron end loss. $E$ may be self-consistently adjusted to give an increased radial ion flux, thereby reducing the heat load on the divertor plates. For floating conducting plates, the electrons adjust across each plate to establish overall ambipolarity consistent with the requirement of no net current flow to each plate. For grounded conducting plates the net electron current to the plates can be balanced by a net ion current to the wall to maintain ambipolarity.

Biasing the divertor plates controls the electric field and the losses to the divertor plates. For example, if $\nabla_{\perp} \Phi$ in the sheath near the plates is large then the $\mathbf{E} \times \mathbf{B} \mathrm{drift}$ is expected to spread the heat load on the divertor plates. The value of the potential gradient is also the determining factor for convection into the private flux region.

In order to determine $\nabla \Phi$ in the divertor region, existing treatments of ambipo- 
larity use $\nabla \cdot \mathbf{J}=0$, and employ pressure balance to determine $\mathbf{J}_{\perp}=\left(c / E^{2}\right) \mathbf{B} \times$ $\nabla \cdot\left(P_{e}+\mathbf{P}_{i}\right)$, where the tensor character of the pressure, coming from ion loss cone effects, may be neglected. Furthermore, the collisional result of Braginskii is invoked to determine $J_{\|}$so that

$$
J_{\|}=\sigma_{\|}\left[E_{\|}+\frac{1}{e N_{e}}\left(\nabla_{\|} P_{e}+\beta_{0} N_{e} \nabla_{\|} T_{e}\right)\right]
$$

with $\beta_{0}=0.71$ and $\sigma_{\|} \approx 1.96 \sigma_{\perp}=1.96 e^{2} N_{e} \tau_{e} / M_{e}$ for $Z=1$. The collisionality decreases going upstream. Using a fluid moment approach of the ion drift kinetic equation this collisional result should be extended to the "plateau" regime. Substantial changes in the determination of the electric field and thus the cross field flows are expected to occur when the collisionality on field lines beyond the separatrix is allowed to vary from the short to the long mean free path limit. The thermal friction term in the paralle' Ohm's law may require a novel flux limiter condition to be worked out when $\nabla_{\|} T_{e}$ becomes too large. Using the appropriate results for $\mathbf{J}_{\perp}$ and $J_{\|}$, these equations can be combined into an elliptic equation for the potential which has to be solved in the domain bounded by the inner and outer divertor plate, the vessel wall, the private flux region, and the plasma boundary (separatrix) between the divertor plasma and the core plasma. Across this boundary the equations determining the electric field may introduce sharp gradients. This can be seen by noting that inside the separatrix the potential is determined by an entirely different set of equations, namely the flux averaged radial projection of Ampere's law coupled to toroidal angular momentum balance. Indeed, the L-H transition experiments on DIII-D exhibit an abrupt jump first of the poloidal and toroidal flow velocities, later of the potential. This suggests that it is primarily the ion flow dynamics which drives the transition, with the electrons and the potential following the ions. The concomitant strongly sheared $\mathbf{E} \times \mathbf{B}$ flows $\left(\frac{\partial}{\partial r} U_{e}\right)$ can result in a change of banana orbit width ("orbit squeezing" and even orbit topology change) and shrinking of the orbit loss cone region.

In high recycling divertors, charge exchange effects are shown to dominate over 
Coulomb collisions as well as ionization and recombination, and conservation of total momentum must be evaluated by retaining the neutrals. Summing the momentum balance equations of all species including the neutrals results in a neutral pressure $p_{n}$, enhancement of $\mathbf{J}_{\perp}$ of the form

$$
\mathbf{J}_{\perp}=\left(c / B^{2}\right) \mathbf{B} \times \nabla\left(P_{e}+P_{i}+p_{n}\right)
$$

which results in an ambipolarity modification. Charge exchange effects as well as inclusion of impurity species) on the parallel flow can also be expected to alter Ohm's law.

Strong poloidal asymmetries of the impurity densities and the potential can be caused by impurity-main ion friction nonlinearities which may explain the inner/outer plate observed heat load symmetry. 


\section{ATOMIC AND MOLECULAR DATA ISSUES}

Atomic and molecular data are needed for power and particle control modelling and diagnostics. Power and particle control includes successful operation of divertors and the maintenance of adequate impurity control.

\section{Hydrogen Collisions}

The major collisions are $\mathrm{H}+p, \mathrm{e}^{-}$. These collisions determine the level of hydrogen recycling and energy losses due to hydrogen radiation and charge exchange. The processes are treated in the codes with collisional radiative models, which are widely used by the modelling groups. The data is generally thought to be adequate, but a comparison of the models would be useful since the data are a very important part of the models.

A second issue is the optical opacity of a high density, low temperature plasma with a high density of neutrals with respect to line radiation. If the divertor plasma is optically thick to hydrogen line radiation, then the behavior of high recycling divertors will be modified, and hydrogen radiation losses would likely be reduced.

In addition, measurement of the particle flux, usually by $\mathrm{H}_{\alpha}$ radiation, in the divertor and edge region is very important in understanding particle transport in those regions. From $\mathrm{H}_{\alpha}$ emission data, the neutral particle source is calculated using a collisional-radiative model. However, cross sections for excitation to and from high-

n states for $\mathrm{H}, \mathrm{H}_{2}$ and $\mathrm{H}_{2}^{+}$are not known to sufficient accuracy. More reliable data for these cross sections are needed to improve the accuracy of particle flux measurements.

\section{Hydrogen Molecules Collisions}

A portion of the plasma in a divertor recycles as molecules. Data for molecular collisions has been tabulated and is used in the models. The major uncertainties are in the fraction of molecules versus atoms desorbed from the wall and divertor plate, and in the role of vibrationally excited hydrogen. Measurements of vibrational states of hydrogen molecules in divertors would be useful. In addition, as mentioned in the 
section on hydrogen collisions, more reliable data for high-n states for interpreting $\mathrm{H}_{\alpha}$ radiation to determine the particle flux would be very useful.

\section{He Collisions}

The major collisions are $\mathrm{He}^{0,+1}+\mathrm{p}, \mathrm{e}^{-}$. Collisional radiative models are widely used, and the rates appear to be in fairly good shape. However, discrepancies may exist for high $\mathrm{n}$ states. Elastic scattering of $\mathrm{He}^{0}$ on $\mathrm{H}+$ is a potentially important process and better data is needed. Work on He data should be part of a program on $\mathrm{H}$ and He recycling.

\section{Impurities}

The major collisions are $\mathrm{H}, \mathrm{He}+\mathrm{A}^{q+} ; \mathrm{A}^{0}+\mathrm{e}^{-} \rightarrow \mathrm{A}^{+} ; \mathrm{A}^{q+}+\mathrm{e}^{-}, \ldots$, including ionization, recombination, excitation, and charge transfer (with neutral atoms) where $\mathrm{A}$ is the impurity atom. One major area where there is still a need for data is the charge transfer cross section for low energy collisions with $\mathrm{H}$ and $\mathrm{H}_{2}$. This is very important for determining the charge state balance for the plasma in the divertor chamber. Quite a bit of data is available for $\mathrm{C}$ and $\mathrm{O}$, but data for higher $\mathrm{Z}$ ions and metals is not available.

Improved radiation emission rates are also needed, especially for low electron temperatures. These are needed to accurately calculate the cooling of divertor plasmas by impurity radiation. Calculations using codes that incorporate meta-stables and improve upon the average ion treatments are needed.

It is also important that the data be collected in a general format that is convenient for use by modelers, i.e. tabular fits for ionization, recombination, excitation, and radiative cooling rates as a function of $\mathrm{n}_{e}, \mathrm{~T}_{e}, \mathrm{n}_{H}$, ionic charge, and $\tau_{\text {residence }}$.

For effective control of impurities, their generation, shielding and transport must all be considered. To address these issues, spatial profiles of impurity neutral particles in low-to-medium ionization stages $q<10$ must be measured and compared with predictions of impurity transport codes. For these measurements and model calculations, accurate a ate coefficients and cross sections for ionization, recombination, 
excitation and charge exchange are required.

\section{Impurity Molecules}

These arise due to the chemical reaction of the plasma constituents and impurities with each other and the plasma facing components such as $\mathrm{C}$ and Be. They include $\mathrm{C}_{n} \mathrm{H}_{m}, \mathrm{H}_{2} \mathrm{O}, \mathrm{CO}, \mathrm{CO}_{2}$, etc. A complete set of rates is needed including reaction rates for ionization, dissociation, and recombination, together with the reaction products and the kinetic distribution of those products for electron and heavy particle collisions. These data are important for modelling the evolution of impurities in the divertor and for diagnosing the behavior of impurities in the divertor.

Although some data collections exist, reliable data for these molecules are still limited. In particular, the cross sections for the production of neutral and charged species due to dissociation are very unreliable. The photon emission rates are also not reliable. One complication is that some of the cross sections involving molecules vary by an order of magnitude depending on their internal energy. The internal states of molecules in a plasma (in particular in the divertor region) should be different from those in laboratory collision experiments so that care is needed for using such cross sections in divertor models and divertor diagnostics. The urgent issues involve collisions of state-selected molecular species, measurement of neutral as well as charged products, including the energy and angular distribution, and a.curate measurements of the photon emission cross sections. Systematic measurements for collision cross sections are urgently needed.

\section{Neutral Collisions}

These include elastic collisions of $\mathrm{H}$ and $\mathrm{H}_{2}$ with the plasma and elastic and inelastic collisions with other neutral atoms. Some data is available in the form of simple scattering potentials. However, better data is needed, particularly at low energies. Elastic scattering of neutral atoms and molecules with the plasma has been included in a number of divertor models, but more accurate and more conveniently tabulated data, is needed. In particular, a convenient tabulation of the differential 
cross section is needed similar to the tabulation of reflection coefficients for neutral particle reflection.

Elastic and inelastic collisions between neutral atoms and molecules are also important for high density divertor operation, particularly the regime where it is hoped to operate divertors for such large, high power machines such as ITER. However, the major limitation in determining the effect of neutral-neutral collisions is the need to develop an ifficient computational technique for computing particle and energy transport when these effects are important.

\section{Vapor Shield Models}

Plasma disruptions can deposit a lot of energy $\left(\sim 10 \mathrm{MJ} / \mathrm{m}^{2}\right)$ on plasma facing components in a short period ( $100 \mu \mathrm{s})$. This can lo $\mathrm{dd}$ to melting and ablation on the surface of the plasma facing component, resulting in a short lifetime of the plasma facing component. The density of the ablated plasma is high $\left(10^{22}-10^{23} m^{-3}\right)$ and the temperature is low $(\sim 1-5 \mathrm{eV})$. This ablated plasm can form a barrier to the further deposition of energy on the plasma facing component by radiating mich of the deposited energy away and by absorbing the heat before it reaches the surface 


\section{IMPURITY TRANSPORT IN THE DIVERTOR REGION}

The divertor is intended to (1) keep high $\mathrm{Z}$ sputtered impurities out of the core plasma by entraining them in the main ion flow to the divertor plates and to (2) use low $\mathrm{Z}$ trace impurities to radiate away excess energy without entering the core plasma.

For the first effect, the impurities influence hydrogenic ion transport in an essential way through parallel friction including the thermal force and the ambipolar condition $\sum_{s} e_{s} \Gamma_{s}=0$ for the fluxes. For the second effect, they may be tenuous enough to allow a test particle (Monte Carlo) treatment such as the DIVIMP code, but this needs to he quantified in each case.

In general, the critical condition for strong impurity hydrogen interaction is $n_{z} Z^{2} / n_{:} \gg \sqrt{m n_{e} / m_{i}} \simeq \frac{1}{60}$. Thus for $Z \sim 6, \frac{n_{z}}{n_{i}} \gg .05 \%$ suffices (to have the impurity-hydrogen fraction $R_{2 i}$ dominate the electron-hydrogen friction $R_{e i}$ driving collisional transport in the pure plasma). [For a review of parallel and perper licular colïsionai impurity transport cf. Hirshman/Sigmar, Nuclear Fusion 211079 (1981).]

In the open field line divertor region the problem of determining the self consistent ambipolar potential gradient is fundamentally altered by the impurity ions, their loss cones, and their transport dynamics. In turn, $\nabla \Phi$ drives all cross field flow (including the connection into the private flux region). This necessitates keeping separate fluid equations for each impurity species and, in principle, even for each charge state (a severe burden for the 2D-fluid codes). Besides the often used simple charge state averaging procedure, there exists a first principles based "reduced charge state" theory (by Hirshman, et al., op. cit., p. 1123) which retains intercharge state friction effects in an exact way such that there remains only one transport equation for each species. This theory was developed for core tokamak plasmas inside the separatrix and must be revisited and adapted for open field line geometry in the divertor where the parallel impurity ion flow velocities are not small compared to their thermal speed as is 
usually assumed in transport theory. These large flow velocities will also require the formulation of a flux limiter condition for the thermal friction force and revisiting the presheath theory including the impurities.

In recent years the "standard" neoclassical impurity theory for the core plasma has been extended by C.T. Hsu and D.J. Sigmar [Plasma Phys. and Contr. Fusion 32 499 (1990)], into a nonlinear regime where not only the inertial terms of the observed strong poloidal flows in the edge and the neutral beam driven toroidal flows are kept but also the ensuing $O(r / R)$ poloidal asymmetries of the impurity density in the flux surface $\bar{n}_{z} / \bar{n}_{z} \sim O(r / R)$ and the ensuing poloidal electric field $\partial \Phi / \partial \theta \sim O(r / R)$. How this field extends across the separatrix is presently not known and needs to be worked out. Furthermore, it has been shown in the Pfirsch-Schlüter region of the core plasma that strong poloidal asymmetries caused by impurities lead to bifurcated transport solutions. Such multiple solutions could be of great interest for a high density gas target-radiating divertor. The observed strong inner plate/outer plate asymmetries in DIII-D should be analyzed from the standpoint of this nonlinear impurity theory. 


\section{POLOIDALLY LOCALIZED POWER DISSIPATION (MARFEs and Dense Gas Targets)}

The divertor heat load can be dissipated over an area larger than that dictated by the radial decay lengths of the plasma density and temperatures if the energy losses in the divertor chamber are completely dominated by radiation and/or charge exchange. Preferably stable, but also possibly oscillating (e.g., ELM-like), theoretical solutions to the problem of strong parallel energy flow into a highly dissipative target would provide guidance for divertor design and experimental studies. There are experimental observations of related problems that could be used as a basis for further development of theoretical models. In the case of strong radiation losses, MARFEs (stable, poloidally localized, toroidal bands of strong radiation just below the density limit in present plasmas) prove that stable solutions do exist under certain conditions. Conditions for their formation and localization would need to be translated to vicinity of $X$-point or divertor region. Plasma flow into a dense neutral gas target requires the inclusion of gas dynamics and atomic and molecular physics. The pellet ablation process (in both magnetically confined plasmas as well as in inertial confinement) is a prime example of a gas shield dramatically reducing the net energy transfer to a solid surface. The existence of strong, high frequency fluctuations in the radiation from the cloud surrounding a pellet in a magnetically confined plasma indicates the existence of benign instabilities. The difficulty in obtaining a stable solution in this case is to minimize backflow to the plasma chamber of both the neutral gas and cold plasma. 


\section{Helium Ash Removal}

He is created at the plasma center, and must then diffusion to the plasma edge, convect down the open field lines to the divertor chamber, recombine at the divertor plate forming neutral He gas which then must be pumped.

\section{Edge Transport}

The He ions need to travel down the open field lines from the plasma edge to the divertor plate. This transport will depend on the friction with the background ions, the thermal force, the anomalous radial transport, and drifts and radial and parallel electric fields. There are predictions that the He will be preferentially transported to the outer portion of the scrape-off layer due to the formation of a vortex like flow of the background hydrogen ions which stagnates the hydrogen flow near the separatrix just below the $X$-point. This leads to enrichment of the He in outer region of the divertor plate increasing the He pumping. All of the effects listed below need to be addressed to accurately determine the He transport to and in the divertor plasmas.

\section{Divertor Transport}

The $\mathrm{He}^{++}$ions recombine on the divertor plate. The fast He atoms then transport through the divertor plasma to the pumping duct. This is a complicated process involving the He atom transport through the divertor plasma with ionization and elastic scattering collisions, and reflection off the pumping duct and divertor chamber walls. There is some indication that He may be preferentially transported to the pumping duct compared to hydrogen because the He has a longer mean free path than hydrogen. The He ionization rate is lower than the $\mathrm{H}$ ionization rate, and He has a very low charge exchange cross section.

The details of He transport in the divertor need to be calculated with an accurate kinetic model which includes all of the relevant atomic and surface processes and the effects of the detailed geometry of the divertor chamber and pumping duct. Typically, Monte Carlo codes are used for this problem. 


\section{Neutral Transport}

Neutral transport calculations are necessary to determine the source terms for density, momentum, and energy due to collisions between the plasma and neutral atoms. These source terms are among the most important determinants of the plasma parameters in the divertor. They are also necessary to determine the gas throughput and pumping efficiency.

Collisions between the neutral atoms and molecules include many processes, including ionization, charge exchange, charge transfer with impurities, excitation, dissociation, and recombination. Collisions between the neutrals and the wall involve reflection, absorption and desorption, and sputtering.

The neutral distributions is dependent on the plasma and vacuum vessel geometry. For many problems, it is inherently a kinetic problem since the mean free path is usually very long in regions of low plasma density. In some cases, a diffusion treatment can be used because the mean free path is very short in regions where the plasma density is high.

Due to kinetic nature of the transport and the number of atomic, molecular, and surface processes involved, Monte Carlo techniques are often used to calculate neutral transport. These codes, such as DEGAS and EIRENE, include all of the relevant processes in realistic geometries, but are computationally slow and have statistical noise. They are therefore difficult to use in fluid codes.

In regions where the plasma density is high and the mean free path is therefore short, diffusion calculations can be used. These are not only much faster computationally, but do not introduce statistical noise. However, they are less accurate, especially near the plasma boundary. Such codes should ideally be multigroup, and include some treatment of momentum transfer as well as ionization sources.

Ideally, a fluid code should have a "suite" of neutral transport models available. These would include:

1. A simple analytic model which would be very fast, and could be used for quick 
parameter surveys. Its accuracy would be quite limited, but it would allow rapid turnaround.

2. A multi-group diffusion model which would be more accurate than the simple analytic models, but which would be fast enough to allow parameter surveys.

3. A detailed Monte Carlo code such as DEGAS which would allow a highly accurate treatment of the neutral transport at the expense of long run times. 


\section{SELECTED PROBLEMS}

We isolate a few problems that we consider of particular significance, many of which are relatively direct, but which could have significant effect on the modelling and understanding of edge and divertor physics. Many other equally significant problems are embedded within the text. We reiterate that at the present time we consider all the problems worthy of some level of effort.

\section{Turbulence}

1. Identification of the dominant transport mechanisms for conventional and advanced (gas target, radiative divertor) regimes.

2. Development of estimates of turbulence-based transport coefficients appropriate for SOL modelling codes. Allocation of heat between electrons and ions.

3. Assessment of effects of turbulence on parallel flows.

4. Development of point models with self-consistent scaling of equilibrium and turbulence.

\section{Sheaths and Boundary Conditions}

1. Model kinetic calculations to improve boundary conditions for the ion flow velocity and energy flux at the sheath boundary, especially for magnetic fields of grazing incidence.

2. Collisional and kinetic effects in the presheath.

3. Non-Maxwellian electron distribution function effects on heat flow in the sheath. 


\section{Electric Field Effects and Related Kinetic Effects}

1. A generalization of parallel Ohm's law to the long mean free path regime including a flux limiter condition for the thermal friction and improved flux limiter expressions for heat flow and viscosity.

2. The determination of the self-consistent potential solution in and outside the separatrix.

3. Matching the potential solution across the separatrix including the Hazeltine "sheath" (at the separatrix) and orbit topology change effects.

4. Calculation of a self-consistent loss cone boundary in the presence of an ambipolar electric field.

5. Development of a theory for the control of the ambipolar electric field through biasing.

6. Comprehensive inclusion of charge exchange momentum loss in determining $\nabla \Phi$

\section{Atomic and Molecular Physics}

1. Improved ionization, excitation, recombination, and charge exchange rates for low temperature plasmas.

2. Better rates for elastic scattering of hydrogen atoms and molecules and helium and low energy neutral-neutral collisions.

3. A complete set of reasonably accurate rates for molecules such as $C_{n} H_{m}, C O_{n}$, $\mathrm{H}_{2} \mathrm{O}$, etc.

4. Better plasma-surface interaction data: 
(a) the reflection of low energy atoms, ions, and molecules, including the effects due to surface roughness,

(b) better sputtering rates, including the synergism of sublimation, physical and chemical sputtering, and surface contaminants,

(c) the desorption rates of atoms, and

(d) the surface recombination rates of molecules.

\section{Impurity Transport}

1. Interaction between impurity ion losses and transport and ambipolar field.

2. Adaption of reduced charge state theory to open field line region for use in $2 \mathrm{D}$ fluid codes.

3. Study of MARFE's and dense gas targets. Development of both theoretical and computational nodels for the problem of plasma flow into a highly dissipative neutral-dominated, radiating target is necessary for design of advanced divertor concepts. MARFE onset, localization and stal ility is a closely related problem that can be used for model verification.

\section{Neutral Transport}

1. The development of computationally simple and accurate neutral diffusion algorithms which can calculate the transport of neutral particles and their momentum energy, and the exchange of the density, momentum and energy between the neutral atoms and the plasma.

2. The development of computational efficient Monte Carlo neutral transport codes which can be used with 2D fluid codes. 


\section{REVIEW PAPERS ON EDGE PHYSICS}

General Review and Overview:

Physics of Plasma Wall Interactions in Controlled Fusion, ed. D. Post and R. Behrisch, F num Publishing Co., 1986.

G. McCraken and P. Stott, Nuclear Fusion 19, 889 (1979).

P.C. Stangeby and G.M. McCracken, Nuclear Fusion 30, 1225 (1990).

Elementary Processes in Hydrogen-Helium Plasmas, by R. Janev, et al., Springer-Verlag, 1987.

R. Langley, et al., Data compendium for plasma surface interactions, Nuclear Fusion Supplement 9117 (1984).

R. Janev, ed., Atomic and plasma-material interaction data for fusion, Nuclear Fusion Supplement 1 (1991).

\section{Conference Proceedings:}

Proceedings of the 3rd International Workshop on Plasma Edge Theory in Fusion Devices, Contributions to Plasma Physics Berlin 32 (1992), 171-498.

Proceedings of the International Conference on Plasma Surface Interactions, Journal to Nuclear Materials, Vols. 176 and 177 (1990); Vols. 162-164 (1989); Vols. 145-147 (1987); Vols. 128 and 129 (1984); Vol. 121 (1984); Vols. 111 and 112 (1982). 

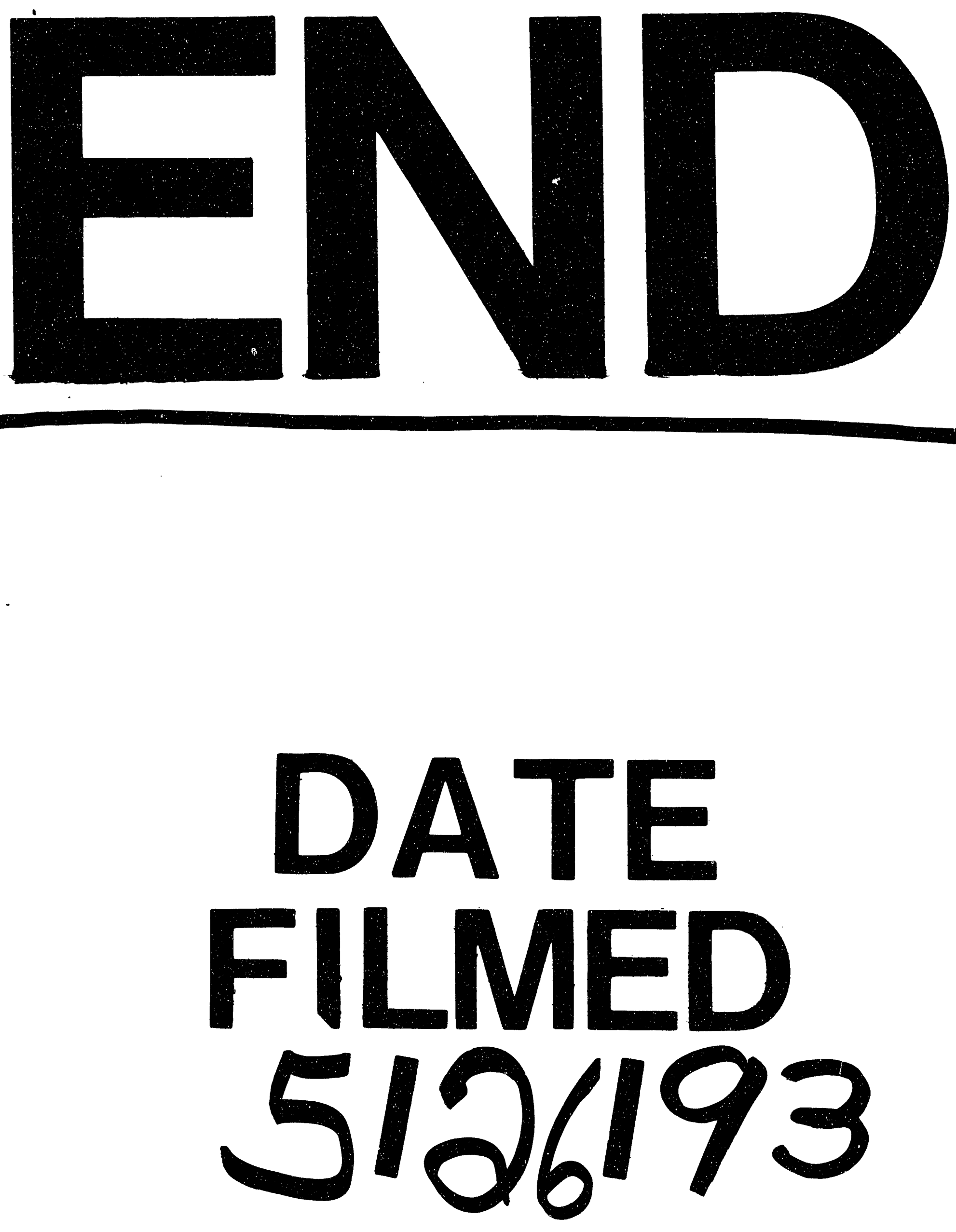
$\pi-\quad \cdots$ 